\title{
Catalytic oxidation of carbon monoxide on a
}

\section{curved Pd crystal: spatial variation of active and poisoning phases in stationary conditions}

Frederik Schiller, ${ }^{\dagger}$ Max Ilyn, ${ }^{\dagger} \ddagger$ Virginia Pérez-Dieste, ${ }^{\top}$ Carlos Escudero, Cristián Huck-Iriart,,${ }^{\S}$ Nerea Ruiz del Arbol, $\|$ Benjamin Hagman, ${ }^{\perp}$ Lindsay R. Merte, ${ }^{\perp}$ Florian Bertram, ${ }^{\perp}$ Mikhail Shipilin, ${ }^{\perp}$ Sara Blomberg, ${ }^{\perp}$ Johan Gustafson, ${ }^{\perp}$ Edvin

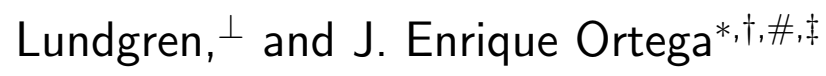

$\dagger$ Centro de Física de Materiales CSIC/UPV-EHU-Materials Physics Center, Manuel Lardizabal 5, 20018-San Sebastian, Spain

$\ddagger$ Donostia International Physics Centre, Paseo Manuel de Lardizabal 4, 20018-San Sebastian, Spain

\ALBA Synchrotron Light Source, Carrer de la Llum 2-26, 08290 Cerdanyola del Vallès, Barcelona, Spain

$\S$ Escuela de Ciencia y Tecnología, Universidad Nacional de San Martín (UNSAM), Campus Miguelete, 25 de Mayo y Francia, 1650 San Martín, Provincia de Buenos Aires, Argentina || Instituto de Ciencia de Materiales CSIC, Madrid, Spain $\perp$ Department of Physics, Lund University, Sweden

\#Departamento Física Aplicada I, Universidad del País Vasco, 20018-San Sebastian, Spain E-mail: enrique.ortega@ehu.es 


\begin{abstract}
Understanding nanoparticle catalysis requires novel approaches in which adjoining crystal orientations can be studied under the same reactive conditions. Here we use a curved palladium crystal and near-ambient pressure X-ray photoemission spectroscopy to characterize chemical species during the catalytic oxidation of $\mathrm{CO}$ in a whole set of surfaces vicinal to the (111) direction simultaneously. By stabilizing the reaction at fixed temperatures around the ignition point we observe a strong variation of the catalytic activity across the curved surface. Such spatial modulation of the reaction stage is straightforwardly mapped through the photoemission signal from active oxygen species and poisoning $\mathrm{CO}$, which are shown to coexist in a transient regime that depends on the vicinal angle. Line-shape analysis and direct comparison with ultra-high vacuum experiments help identifying and quantifying all such surface species, allowing us to reveal the presence of surface oxides during reaction ignition and cooling-off.
\end{abstract}

\title{
Introduction
}

The $\mathrm{CO}$ oxidation $\left(2 \mathrm{CO}+\mathrm{O}_{2} \rightarrow \mathrm{CO}_{2}\right.$ ) on platinum group metal surfaces, such as palladium, has been intensively studied during decades, due to its enormous technological impact, and also as a model heterogeneous gas/surface catalytic reaction. ${ }^{1}$ In the early times, ${ }^{2,3}$ Surface Science experiments carried out in Ultrahigh Vacuum (UHV) played an essential role in the basic understanding of the $\mathrm{CO}$ oxidation process, but a much deeper atomic-scale insight is being lately gained through surface sensitive techniques that operate at millibar and bar pressures, such as high-pressure Scanning Tunneling Microscopy (STM), ${ }^{4,5}$ Near-Ambient Pressure X-ray photoemission spectroscopy (NAP-XPS), ${ }^{6-8}$ infrared reflection absorption spectroscopy ${ }^{9-13}$ and high-energy surface X-ray diffraction. ${ }^{14-23}$ All these studies agree in the fundamental picture, namely the abrupt transition at the "ignition" temperature, from the low-temperature oxidation stage, when CO covers (or "poisons") the catalytic surface, to the high-temperature activity stage, when the CO-poisoning layer is displaced by chemisorbed 
oxygen, preceding the build-up, first, of two-dimensional (2D) surface oxides, and last, of bulk oxides. Yet new experiments and approaches are needed to properly identify the driving mechanisms and the active sites that trigger the passage to the active stage.

In the case of $\mathrm{Pd}$, the $\mathrm{CO}$ oxidation reaction has been investigated on a variety of high and low symmetry crystal surfaces under reaction conditions. ${ }^{4-8,10,13,21-28}$ This will help to separately understand the catalytic performance at crystal planes that shape technologically relevant nanoparticles. However, given the variety of reaction parameters and experimental constraints, comparative in-situ investigations among different surface orientations would be better suited. In the end, all facets in nanocrystals coexist in a reduced space, and are assumed to undergo simultaneous chemical and structural transformations during catalytic reactions. ${ }^{29}$ Therefore, more realistic model systems are needed to fill this gap between individual crystallographic planes and nanoparticles. ${ }^{30,31}$ In this context, a cylindrical crystal sample can be designed to contain a selected variety of surface planes, which can be exposed to the same reacting conditions, and on which, e.g., the full analytical power of XPS can be preserved. ${ }^{32}$ The cylindrical surface approach that is used here is schematically depicted in Fig. 1a for a Pd crystal. The curved direction of the sample spans the complete set $\left(\Delta \alpha= \pm 16^{\circ}\right.$ ) of vicinal orientations for the two type of close-packed atomic steps (called A and B) around the (111) symmetry direction. Since the radius of curvature of the sample (16 $\mathrm{mm})$ is much larger than the X-ray light spot $(20 \mu \mathrm{m})$, separate crystallographic planes can be sequentially probed by macroscopic sample scanning ( $0.5 \mathrm{~mm}$ steps). In contrast to the full-cylinder approach tested earlier in catalysis studies ${ }^{33}$ the present sample design makes it simple to selectively probe all vicinal orientations with a standard synchrotron photon beam, without having to modify the sample manipulator or the NAP-XPS setup.

By scanning the Pd cylindrical crystal of Fig. 1a with the X-ray beam in the NAP-XPS station of the ALBA synchrotron (Barcelona, Spain), we have been able to map surface and gas phases at all close-packed vicinal $\operatorname{Pd}(111)$ planes during the catalytic oxidation of carbon monoxide. The temperature-dependent hysteretic process around the ignition 
temperature (arrows indicate heating and cooling) is schematically described in Fig. 1b. In this work we establish stationary conditions of gas flow, pressure and sample temperature during the transition from the low- to the high-activity stages (dashed lines in Fig. 1b), such as to accurately determine surface phases at each reaction stage and as a function of the crystallographic orientation. As it occurs in other $\mathrm{Pd}$ and transition metal surfaces, ${ }^{8,34}$ we find that the low-activity stage is linked to the presence of a poisoning CO layer, which is replaced in the high-activity phase by an active layer containing both chemisorbed $\mathrm{O}$ and metal oxides, in variable proportions across the curved surface. We also find that the transition is faster for $\{111\}$-like (B-type) with respect to $\{100\}$-like (A-type) vicinals, and also with respect to the $\operatorname{Pd}(111)$ surface, for which a $\sim 45 \mathrm{~K}$ transient state is found. The latter allows the surprising coexistence of both poisoning $\mathrm{CO}$ and active $\mathrm{O}$ species on the surface, both during ignition and cooling-off.

\section{Experimental}

\section{Curved sample processing in Ultra High Vacuum}

The curved Pd(111) surface (Bihurcrystal Ltd., Spain) is obtained by mechanical erosion of a flat $\operatorname{Pd}(111)$ crystal, followed by mechanical polishing down to $0.25 \mu \mathrm{m}$ grinding. In UHV, the sample was extensively treated by alternating sputtering (1 keV Ar ion beam) and thermal flashing $(1200 \mathrm{~K})$. To remove any possible $\mathrm{C}$ segregation, the annealing cycle was periodically performed in the presence of $\mathrm{O}_{2}\left(1 \times 10^{-8}\right.$ mbar, 10 minutes $)$. Sputtering was carried out at $45^{\circ}$ incidence and with the scattering plane of the Ar ions parallel to the surface steps.

\section{Near-Ambient pressure X-ray Photoelectron Spectroscopy setup}

The NAP-XPS spectra were measured at the NAPP-CIRCE beamline of the ALBA synchrotron light source (Barcelona, Spain) with a PHOIBOS 150 NAP analyzer from SPECS. 
(a)

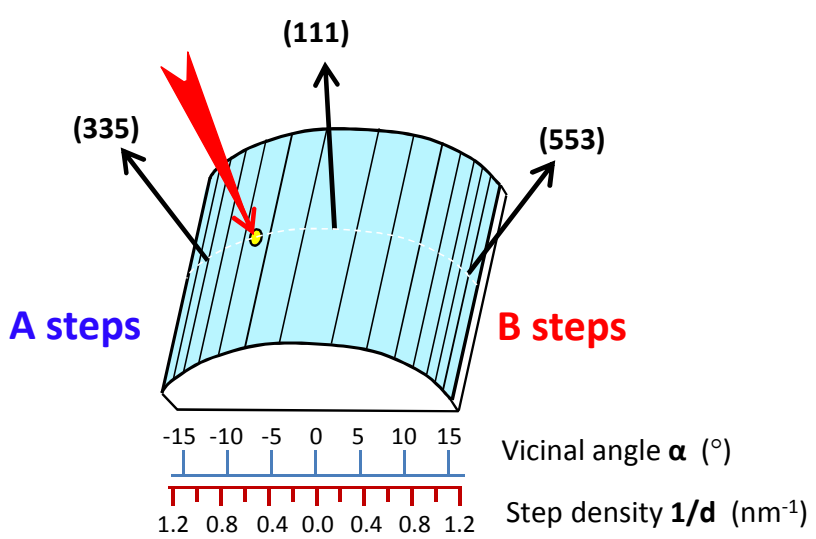

(b)

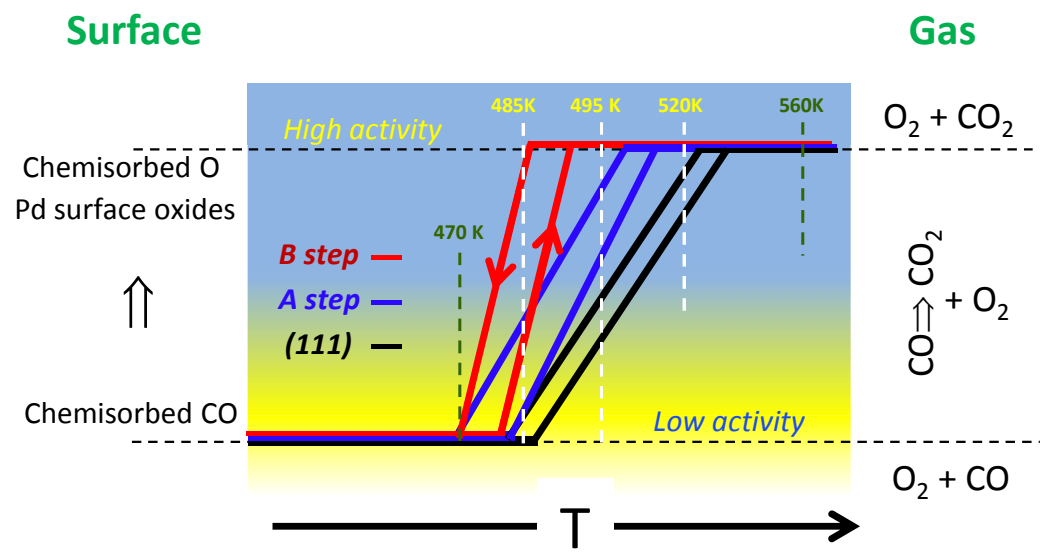

Figure 1: Catalytic oxidation of CO on a curved Pd crystal. (a) Sketch of the curved $\operatorname{Pd}(111)$ sample featuring A-type and B-type vicinal surfaces around the center of the crystal, which is oriented along the high-symmetry (111) direction. (b) Schematic diagram for the hysteretic transition between the high- and low-activity stages of the catalytic oxidation of $\mathrm{CO}$ on $\mathrm{Pd}(111)$, and on stepped A-type and B-type surfaces. The ignition of the catalytic reaction sets in faster and at lower temperature in vicinal surfaces with B-type steps, as compared to A-type steps and the (111) plane.

The analyzer is equipped with four differentially pumped stages connected through small apertures. A set of electrostatic lenses focuses the electrons through the apertures to maximize transmission. ${ }^{35,36}$ Such a set-up allows the detector to be kept in UHV while the sample is at a maximum pressure of 20 mbar. The instrumental, beamline plus analyzer, energy resolution during the experiments was better than $0.3 \mathrm{eV}$. The beam spot size at the sample is $100 \times 20 \mu^{2}$ (horizontal $\times$ vertical), with the horizontal axis oriented parallel to surface steps, thereby allowing to probe vicinal angles with a small spread of $\Delta \alpha<0.05^{\circ}$. The 
light incidence was normal to the (111) surface. For XPS scans over the curved surface, the sample was rigidly shifted in front of the beam, resulting in a linearly variable emission angle from $\sim 55^{\circ}$ at the A side of the crystal to $\sim 25^{\circ}$ at the $\mathrm{B}$ side of the crystal. This did not affect the surface sensitivity, bat caused a steady $30 \%$ intensity decrease of gas phase core-levels across the curved sample, from A to B, as evidenced in Figs. 2 and 4.

\section{High resolution Ultrahigh Vacuum X-ray Photoelectron Spectroscopy}

High-resolution Photoemission spectroscopy with the substrate exposed to CO and molecular oxygen under Ultra-High Vacuum conditions was carried out at I311 beamline at MaxII in Lund, Sweden. To achieve accurate scans across the curved sample, the light spot size was reduced to $100 \mu \mathrm{m}$ by means of the exit slit, and hence the vicinal angle spread was $\Delta \alpha<0.25^{\circ}$. XPS $\alpha$-scans were taken with the sample temperature kept within the 150-200 K range. The photon energy was varied to optimize the cross section, the surface sensitivity and, in the case of the $\mathrm{Pd} 3 d$, the ability to resolve surface and bulk emission lines across the whole curved surface. Gases were dosed while holding the sample at room temperature $\left(0.2 \mathrm{~L} \mathrm{CO}_{2}, 10 \mathrm{~L} \mathrm{O}_{2}\right)$ and at $380 \mathrm{~K}\left(10 \mathrm{~L} \mathrm{CO}_{2}\right)$.

\section{Line fitting of XPS spectra}

Fitting of all XPS spectra is performed using Donjiac- Šunjic lines ${ }^{37,38}$ convoluted with Gaussian functions. Fitting of the high-pressure data is preceded by the previous analysis of the $\mathrm{Pd} 3 p_{3 / 2}, 3 d_{5 / 2}, \mathrm{C} 1 s$, and $\mathrm{O} 1 s$ spectra from clean and $\mathrm{CO}$ and $\mathrm{O}_{2}$ chemisorbed surfaces acquired in UHV conditions (see also supplementary information). For such process we include $0.2 \mathrm{~L}$ and $10 \mathrm{~L} \mathrm{CO}$, as well as $10 \mathrm{~L} \mathrm{O}_{2}$ data. These UHV experiments set the initial energy and line-shape values for bulk and surface components of $\mathrm{Pd}$, and chemisorbed $\mathrm{CO}$ and $\mathrm{O}$ peaks during the fitting of the high-pressure spectra. The number of peaks for adsorbed species used in UHV fits is the minimum needed for a good result. At this point, we also rely on literature data to identify different chemisorbed components. ${ }^{24,39,40}$ Once the 
initial set of peaks is established for clean and $\mathrm{CO}$ and $\mathrm{O}_{2}$ chemisorbed species, we turn to the fitting of the spectra for the CO oxidation experiment. The starting point is the (111) surface, and then we consider the A- and B-edges of the sample, trying to keep constant line shapes and positions (energy shifts permitted below $100 \mathrm{meV}$ ) of all individual peaks, only allowing variations in peak intensity. In NAP-XPS spectra, we introduce additional lines for the $\mathrm{CO}, \mathrm{O}_{2}$, and $\mathrm{CO}_{2}$ gas phase peaks.

\section{Results and discussion}

$\mathrm{CO}$ and $\mathrm{O}_{2}$ gases were introduced in the XPS chamber in a constant flow regime of 0.15 $\mathrm{ml} / \mathrm{min}$, up to a stable 0.3:0.3 mbar mixture, and with the Pd curved sample kept at 370 K. Then the temperature was stepwise increased until the whole sample reached the high activity stage at $560 \mathrm{~K}$. In a second process, the temperature was stepwise decreased down to $470 \mathrm{~K}$, such as to completely cool off the catalytic reaction in the cylindrical sample. In Fig. 2a-c we examine the steady distribution of surface chemical phases across the curved surface at $485 \mathrm{~K}$ during the cooling-off (see the corresponding dashed line in Fig. 1b). C $1 s$ (Fig. 2a) and O $1 s$ (Fig. 2b) spectra are acquired at seventeen different points of the surface, in $\Delta \alpha=2^{\circ}$ vicinal angle steps, and their intensity is displayed in a color scale. In the $\mathrm{C} 1 \mathrm{~s}$ spectrum we observe two features, the emission at higher binding energy, originating from the $\mathrm{C} 1 s$ core level of $\mathrm{CO}_{2}$ gas between the analyzer nozzle and the sample, and a sharp signal from the CO chemisorbed phase at $285.7 \mathrm{eV}$. The latter reaches its maximum intensity at the center of the sample $[\operatorname{Pd}(111)$ face], and rapidly decreases towards the stepped edges. The $\mathrm{O} 1 \mathrm{~s}$ emission from chemisorbed and oxide species appears as a shoulder in the low binding energy side of $\mathrm{Pd} 3 p_{3 / 2}$ peak, with the reverse intensity variation: it is maximum at the densely stepped edges and vanishes at the (111) plane. Therefore, both Figs. $2 \mathbf{a}$ and $2 \mathbf{b}$ demonstrate that at $485 \mathrm{~K}$ the catalytic oxidation of CO switches off at the (111) surface, but remains ignited at highly stepped crystal planes, with B-type steps being more active 

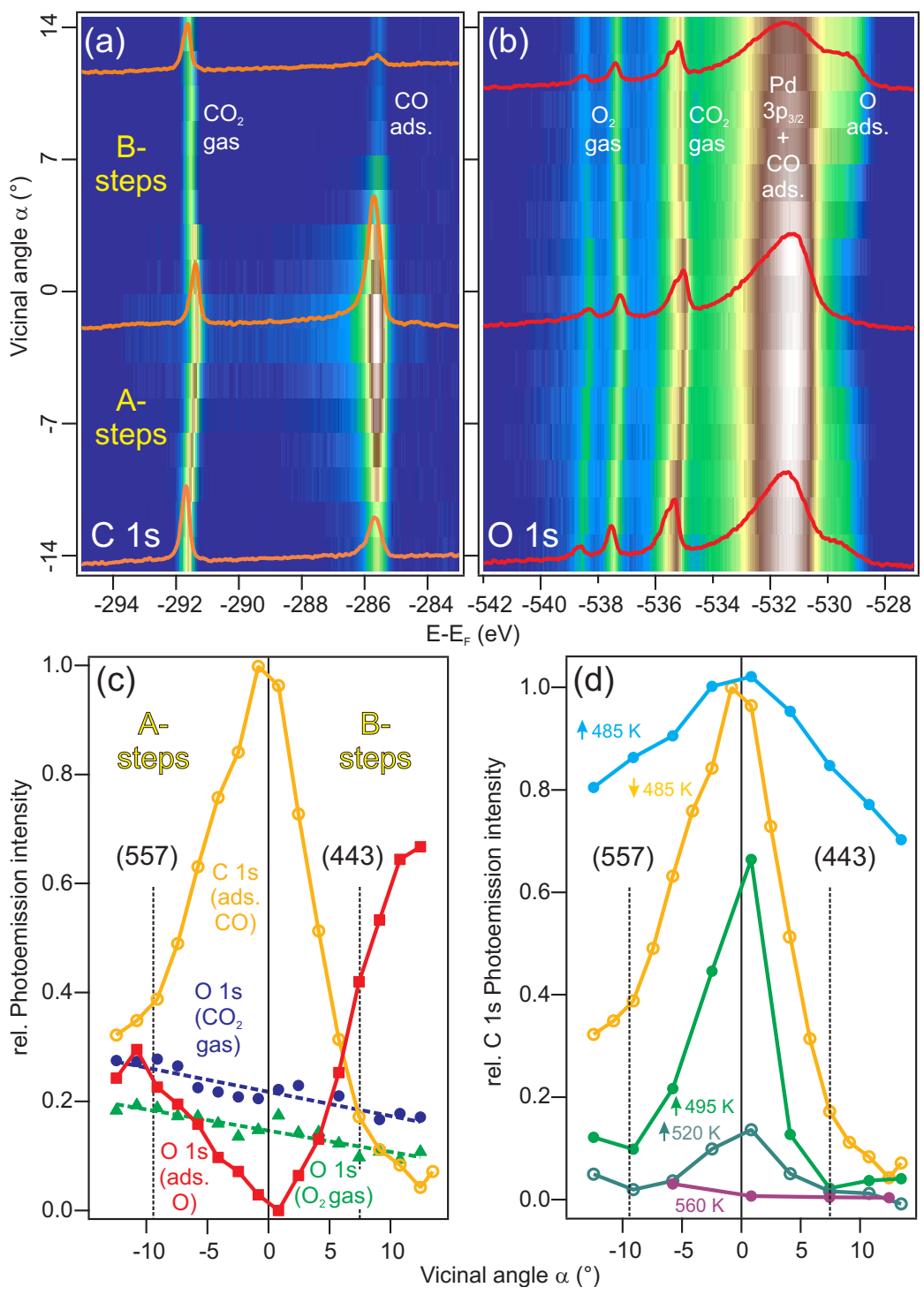

Figure 2: XPS maps of stationary states for a 0.3:0.3 mbar $\mathrm{CO}: \mathrm{O}_{2}$ gas mixture. Intensity plots of (a) $\mathrm{C} 1 s$ and (b) $\mathrm{O} 1 s$ regions across the curved surface at $485 \mathrm{~K}$, during reaction cooling-off. Individual XPS spectra at the center and the densely stepped A and $\mathrm{B}$ edges appear overlaid. In the $\mathrm{C} 1 s$ spectrum, the emission at $285.7 \mathrm{eV}$ corresponds to chemisorbed $\mathrm{CO}$, and its strong variation indicates that the reaction is poisoned at the sample center, becoming progressively more active towards the A-side, and fully active at the B-edge of the sample. In the $\mathrm{O} 1 s$ spectrum in (b) active oxygen-rich species appear as a low-binding energy shoulder of the $\mathrm{Pd} 3 p_{3 / 2}$ peak. (c) $\alpha$-dependent XPS intensity of chemisorbed $\mathrm{CO}(\mathrm{C} 1 s)$ and O-rich $(\mathrm{O} 1 s)$ surface species, and $\mathrm{CO}_{2}$ and $\mathrm{O}_{2}(\mathrm{O} 1 s)$ in the gas phase. Abrupt changes of slope occur in both A- and B-sides, at the marked (557) and (443) planes, respectively. Data are determined from line-fit analysis of individual spectra. (d) $\alpha$-dependent XPS intensity of chemisorbed CO (C $1 s$ ) at different temperatures during reaction cooling-off (downward arrow) and ignition (upward arrow). 
than A-type steps. ${ }^{41}$

In Fig. 2c we represent the $\mathrm{C} 1 s$ and $\mathrm{O} 1 s$ peak intensities as a function of the vicinal angle $\alpha$, together with the $\mathrm{O} 1 s$ intensity from $\mathrm{O}_{2}$ and $\mathrm{CO}_{2}$ in the gas phase. Data points are obtained after line-fitting individual spectra from Figs. 1a and b (see Supplementary Information (SI), Fig. 3 and Methods). At the B-step side, the adsorbed-CO intensity linearly decreases from the (111) direction up to a critical $\alpha \sim 7.5^{\circ}$, beyond which the intensity drop is also linear, but with a reduced slope. As indicated in the figure, such vicinal angle roughly corresponds to the (443) crystal plane. In the A side, a somewhat less-marked change of slope occurs at the (557) surface direction, namely at $\alpha \sim 10^{\circ}$. Notably, the $\mathrm{O} 1 \mathrm{~s}$ intensity exhibits an almost complementary $\alpha$-dependent variation to that of the $\mathrm{C} 1 s$ from chemisorbed $\mathrm{CO}$, and both contrast with the almost constant intensity from $\mathrm{CO}_{2}$ and $\mathrm{O}_{2}$ gas, which suggests a rapid gas diffusion and homogenization in front of the surface. ${ }^{42}$ Finally we note the binding energy variation as a function of $\alpha$ observed in gas peaks of Figs. 2a and $\mathbf{b}$, which mirrors the local work-function at the curved surface. Notably, the sign and magnitude of the shift (up to $0.4 \mathrm{eV}$ ) are similar to the work-function difference between flat and stepped surfaces covered with CO, ${ }^{43,44}$ suggesting that the local substrate plane rather than the difference in the chemisorbed phase is the main reason for this $\alpha$-dependent shift.

XPS scans under stationary conditions have been taken at the temperatures marked in Fig. 1b, during catalytic activation (upward arrow) and cooling-off (downward arrow). In Fig. 2d we show the $\mathrm{C} 1 s$ intensity variation for chemisorbed $\mathrm{CO}$ at such different temperatures, revealing the strong asymmetric response of the surface during the ignition process. Data are normalized to the maximum CO signal detected, which corresponds to the (111) plane in the low-activity phase. The low-activity stage extends to the whole sample at 485K previous to ignition. At this temperature, the peak intensity decreases linearly from the (111) center down to $20 \%$ and $35 \%$ at A and B stepped areas, respectively. The same trend is found for the CO saturation coverage in Ultra High Vacuum (UHV) at $300 \mathrm{~K}$ (see Supplementary Information in Ref. 41), reflecting a decreasing CO chemisorption energy 
for increasing $\alpha$, and for B-type versus A-type vicinal surfaces. The high-activity stage is reached in the whole sample at $560 \mathrm{~K}$, where no chemisorbed $\mathrm{CO}$ signal is detected. We may also compare curves in Fig. $2 \mathbf{d}$ at fixed sample points, such as to assess the temperature span of the transient stage at each surface plane. In the B side of the crystal, and beyond the (443) plane, it is striking to observe that the high-activity stage is kept down to $485 \mathrm{~K}$, while the CO poisoning of the surface is completed at $470 \mathrm{~K}$. Such abrupt transition from the high-activity to the low-activity stage in the B-edge of the sample contrasts to the more gradual decay of the catalytic activity in the (111) surface at the centre of the crystal. In fact, at the (111) surface CO poisoning already starts at 520K, and steadily increases and saturates at $485 \mathrm{~K}$. The A-step side appears as an intermediate case, with a linear increase of $\mathrm{CO}$ from a residual amount at $520 \mathrm{~K}$, to a $20 \%$ of the maximum signal at $495 \mathrm{~K}$, a $40 \%$ at $485 \mathrm{~K}$, and a complete $\mathrm{CO}$ saturation at $470 \mathrm{~K}$.

The XPS scans of Fig. 2 prove the existence of a small temperature range $\Delta T$ during ignition and cooling-off, at which stable mixtures of poisoning and active surface species can be attained. Thus, the commonly assumed view of the $\mathrm{CO}$ oxidation as an autocatalytic process, or "thermal explosion", does not hold in the present case, particularly at the (111) center of the crystal. In fact, from the vertical cut in Fig. $2 \mathbf{d}$ at $\alpha=0$ one can crudely determine $\Delta T \sim 45 \mathrm{~K}$ at the $\operatorname{Pd}(111)$ plane. An even wider transient state has been recently reported for $\operatorname{Ir}(111)(\Delta T \sim 75 \mathrm{~K}),{ }^{45}$ in contrast to the sharp transition $(\Delta T<$ $20 \mathrm{~K}$ ) that characterizes $\mathrm{Pt}(111)$. As discussed for the $\operatorname{Ir}(111)$ case, the stability of the mixed $\mathrm{CO}-\mathrm{O}$ chemisorbed layer is due to the high adsorption energy of $\mathrm{CO}$ and oxygen, which allow them overcome their mutual repulsive interaction. From the point of view of the $\mathrm{CO}$ adsorption energy, $\mathrm{Pd}(111)$ is an intermediate case between $\operatorname{Ir}(111)$ (high adsorption energy) and $\mathrm{Pt}(111)$ (low adsorption energy), as shown in thermal desorption experiments and systematic calculations. ${ }^{46-50}$ However, in vicinal $\mathrm{Pd}(111)$ the $\mathrm{CO}$ adsorption energy decreases as a function of $\alpha$. This indeed explains the decreasing ignition temperature across vicinal planes observed in the curved Pd surface, ${ }^{41}$ but could also be the reason for 

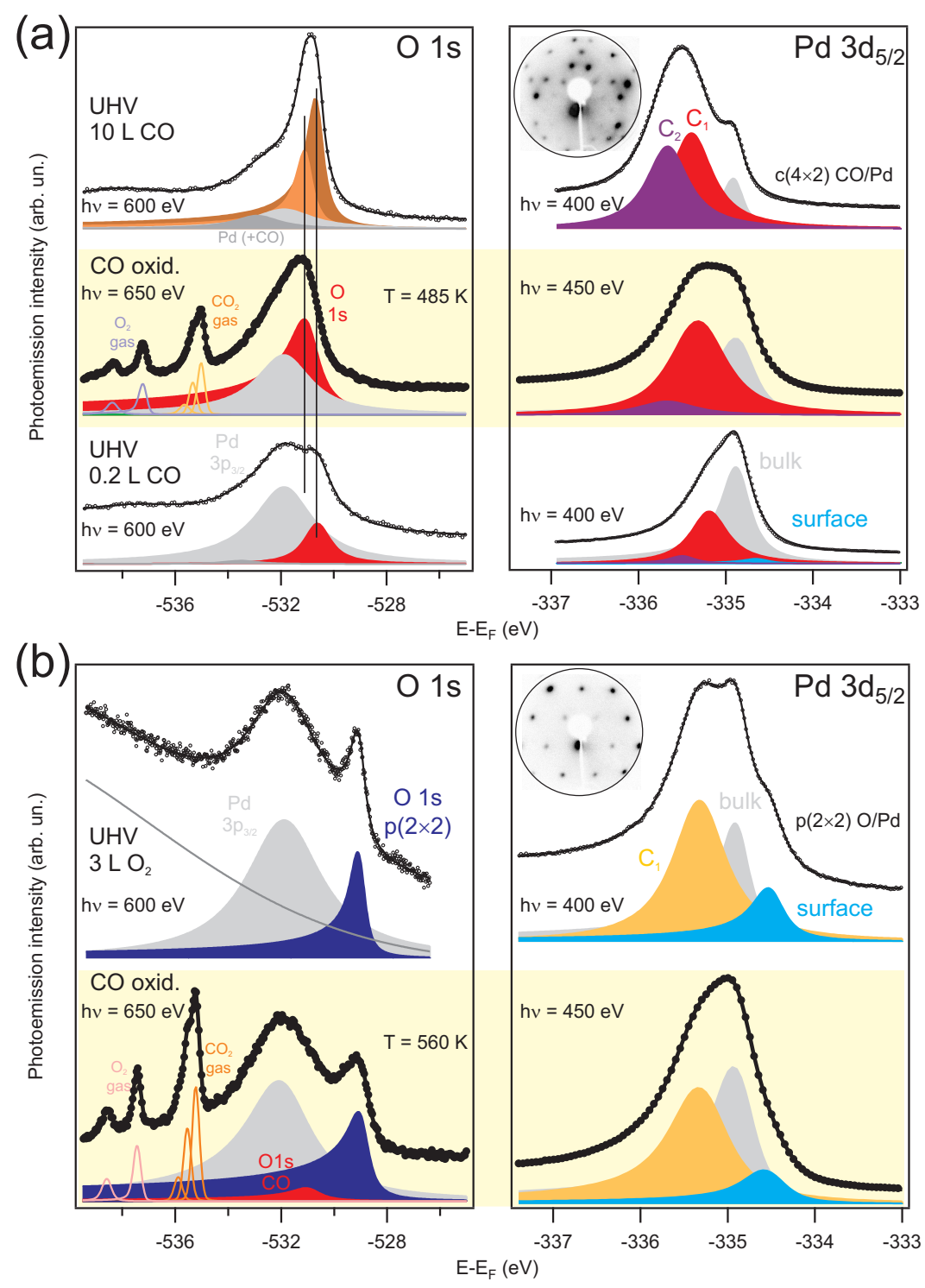

Figure 3: Low-activity and high-activity surface phases compared to chemisorbed phases in UHV (a) Comparative analysis of the $\mathrm{O} 1 s$ and $\mathrm{Pd} 3 d_{5 / 2}$ spectra between the poisoned phase at NAP (yellow background) and the CO chemisorbed $\mathrm{Pd}(1111)$ surface in UHV for $0.2 \mathrm{~L}$ and $10 \mathrm{~L}$ CO exposures (see SI for UHV peak labeling and discussion). (b) Comparison of $\mathrm{O} 1 \mathrm{~s}$ and $\mathrm{Pd} 3 d_{5 / 2}$ between the active reaction stage (yellow background) and the $p(2 \times 2)$ oxygen-chemisorbed phase in UHV. LEED patterns in the insets prove the presence of the $c(4 \times 2)$-CO $(10 \mathrm{~L} \mathrm{CO})$ and the $p(2 \times 2)$-O chemisorbed phases in UHV (see SI for detailed LEED images).

the behavior demonstrated in Fig. 2 (d), that is, the existence of a transient active state in the (111) center, as opposed to the sharp low-to-high activity transition at the densely stepped B-edge. 
To properly identify and quantify phases at the active and poisoned stages we perform a thorough and consistent line fit analysis of individual NAP-XPS and UHV spectra together, as shown in Fig. 3 (see Methods and Ref. 38). The UHV environment allows both the optimal performance of XPS and the parallel structural LEED analysis, ${ }^{39,40,51}$ providing the accurate quantitative evaluation of surface chemical species in NAP-XPS. In Fig. 3 the different panels show this comparison for the $\mathrm{Pd} 3 d_{5 / 2}$ and $\mathrm{O} 1 s$ peaks. All UHV and NAP spectra correspond to the $\operatorname{Pd}(111)$ plane at the center of the crystal (see SI for a deeper discussion on UHV spectra). The low-activity stage must be compared with the spectra acquired after UHV exposure of 0.2 Langmuir (L) and $10 \mathrm{~L}$ of $\mathrm{CO}$ gas. The peaks in the poisoned phase during the $\mathrm{CO}$ oxidation at $485 \mathrm{~K}$ are equivalent (energy, line-shape) to the UHV ones, but the intensity ratios of the different lines indicate an intermediate CO coverage. In the $10 \mathrm{~L}$ spectra at $\mathrm{UHV}$, both $\mathrm{O} 1 s$ and $\mathrm{Pd} 3 d_{5 / 2}$ exhibit the characteristic high-binding energy components of highly-dense chemisorbed CO phases $[>0.5$ monolayers $(\mathrm{ML})$, which indicate a variety of chemisorption sites. ${ }^{39,40,51}$ These are absent in the $\mathrm{O} 1 s$ and $\mathrm{Pd} 3 d_{5 / 2}$ spectra of the poisoned phase during the oxidation reaction. In contrast, for the $0.2 \mathrm{~L}$ exposure in UHV the $\mathrm{Pd} 3 d_{5 / 2}$ peak exhibits a residual surface emission that is not observed in the high-pressure poisoned phase. Judging from the $\mathrm{O} 1 s$ intensity, as well as from the $\mathrm{Pd} 3 d_{5 / 2}$ emission of CO-adsorbed $\mathrm{Pd}$ atoms, we conclude that the CO coverage in the low-activity phase at $485 \mathrm{~K}$ lies around $0.3 \mathrm{ML}$. The active phase at $560 \mathrm{~K}$ is more simple to identify and quantify. In contrast to the relevant presence of oxides under highly oxidative $\mathrm{CO}: \mathrm{O}$ mixtures ${ }^{24}$ under the present 1:1 regime the active phase entirely corresponds to a pure chemisorbed $\mathrm{O}$ (chem-O) layer. This is readily judged from both, the characteristic $\mathrm{O}$ $1 s$ line, and the residual emission from bare $\mathrm{Pd}$ atoms at the low-energy side of the $\mathrm{Pd} 3 d_{5 / 2}$ peak. The intensity ratio with respect to the bulk $\mathrm{Pd} 3 d_{5 / 2}$ and $\mathrm{Pd} 3 p_{3 / 2}$, as well as the residual contribution from $\mathrm{CO}$, indicate that the $\mathrm{O}$ coverage remains slightly below the 0.25 ML of the fully-saturated $p(2 \times 2)$ chem-O layer. 

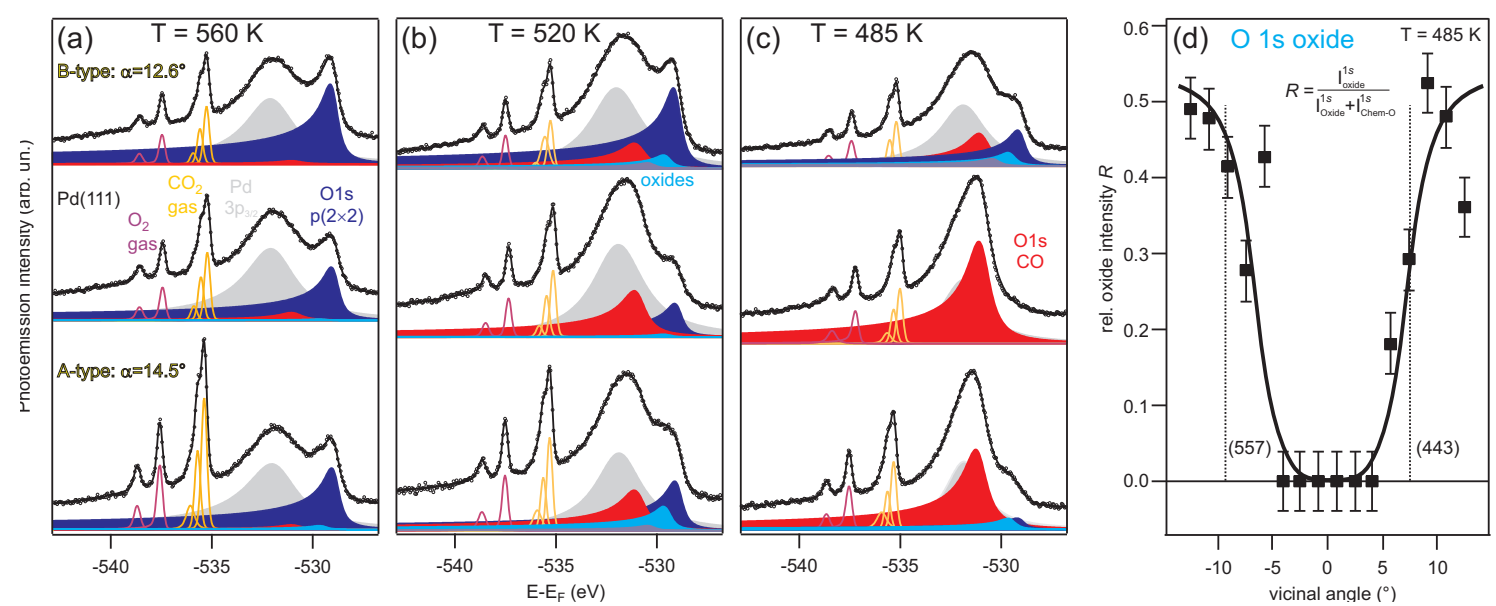

Figure 4: Active surface phases. Line-fit analysis of the O $1 s$ spectra taken at (a) 560 $\mathrm{K}$ (b) $520 \mathrm{~K}$ and (c) $485 \mathrm{~K}$ during reaction cooling-off, at the indicated sample positions: A-type edge (lower spectra), B-type edge (upper spectra) and (111) plane (center). The substrate signal is shaded in gray, chemisorbed CO in red, and the active oxygen-related species in dark blue (chemisorbed oxygen) and light blue (surface and subsurface oxides). The former dominates the spectrum at the fully-active stage at $560 \mathrm{~K}$, whereas the latter build up at intermediate temperatures and at the highly stepped edges of the sample. (d) Relative oxide intensity as a function of the vicinal angle. Data correspond to the stationary state at $485 \mathrm{~K}$ during cooling-off (see Fig. 2).

To gain a further insight into the $\alpha$-angle dependence of the catalytic reaction reflected in Fig. 2, we inspect the temperature evolution of surface phases in individual XPS spectra at three different crystal planes. The CO chemisorbed phase, characterized by the $\mathrm{C} 1 s$ emission shown in Fig. 2a, shows no appreciable shifts or satellites, pointing to a rather homogeneous $\mathrm{CO}$ chemisorption site at all vicinal angles. The $\mathrm{O} 1 \mathrm{~s}$ spectra in Fig. 4a correspond to three characteristic sample points (A edge, B edge, and center) and temperatures during the reaction cooling-off. At $560 \mathrm{~K}$, the chem-O layer (dark blue) is present in every surface plane, with the highest concentration at the B-side and the lowest at the (111) plane, as judged from the $\mathrm{O} 1 s$ intensity relative to that of the $\mathrm{Pd} 3 p_{3 / 2}$ (green). However, the XPS spectra at the intermediate $520 \mathrm{~K}$ shows a significant presence of $\mathrm{O} 1 \mathrm{~s}$ emission in between the $\mathrm{CO}$ and the chem-O species. To explain such contribution, we introduce extra peaks in the fit at around $530 \mathrm{eV}$ binding energy (blue lines), which can be assigned to surface/subsurface Pd oxides featuring highly coordinated oxygen atoms. ${ }^{24,40,52}$ Such oxide emission reveals larger 
intensity in the densely stepped A and B sides, and zero intensity at the $\operatorname{Pd}(111)$ surface. $\mathrm{Pd}$ surface/subsurface oxides of increasing O-coordination are known to sequentially appear on $\mathrm{Pd}(111)$ exposed to $\mathrm{O}_{2} \cdot{ }^{52,53}$ This is the case during reaction ignition (see Fig. S2 in SI), where the chem-O species is the first active phase to arise before the surface oxide is formed. However, in metastable $\mathrm{O}_{2}$-rich atmospheres $\mathrm{Pd}$ oxides are stable only at low-temperature, while at higher temperature a single chem-O layer can stay. ${ }^{30,53}$ This equilibrium of oxygenrelated phases manifests during reaction cooling-off: at $560 \mathrm{~K}$ chem-O is the only active species on the surface, at $520 \mathrm{~K}$ the oxide emerges, and at $485 \mathrm{~K}$ the chem-O intensity is largely suppressed, while the surface oxide emission remains.

The relative contribution of chem-O and surface oxide species to the total $\mathrm{O} 1 \mathrm{~s}$ signal (oxide+chem-O) abruptly changes across the curved crystal at the critical $\alpha$ angles, as shown in Fig. 4d. The data correspond to the stationary state at $485 \mathrm{~K}$ during the cooling-off cycle (see the corresponding total oxygen intensity in Fig. 2c). No oxide is detected within a relatively wide $\Delta \alpha= \pm 5^{\circ}$ range around the (111) direction, and it steeply grows beyond that vicinal angle in both $\mathrm{A}$ and $\mathrm{B}$ sides. The absolute amount of oxide is notably higher at the $\mathrm{B}$ side, where at $485 \mathrm{~K}$ the $\mathrm{CO}$ oxidation reaction remains active during cooling-off. Since at $485 \mathrm{~K}$ the reaction has not started in the ignition cycle, this indicates that the hysteresis is caused by the ultimate reduction of these surface/subsurface $\mathrm{Pd}$ oxides, which build up in the presence of chem-O during the active phase. Chem-O is likely inducing substrate faceting of highly dense vicinal planes at the early ignition stage. ${ }^{15,19,23}$ Therefore, one could conversely speculate that the ability to develop surface/subsurface oxide facets, which certainly increases beyond a critical step density, explains the peculiar spatial modulation of the catalytic activity on the Pd curved surface that is mirrored in Fig. $2 \mathbf{d}$. 


\section{Summary}

In summary, the full power of near-ambient pressure XPS to identify surface chemical species in reaction conditions, added to the robustness of the curved surface approach, allows a consistent description of the surface chemistry during the catalytic oxidation of $\mathrm{CO}$ on $\mathrm{Pd}(111)$ and its vicinal planes. We find that active and poisoned phases above and below the ignition temperature are equivalent to UHV-related phases in all crystallographic planes. However, the strong spatial modulation of the catalytic activity at constant temperature during ignition demonstrates that the reaction is activated in the local plane, with clear A-B asymmetry. One can in fact freeze ignition or cooling-off processes to observe the coexistence of poisoned and active surface species within a small temperature range that depends on the vicinal angle. Yet the passage from the low- to the high -activity stage is rather abrupt for B-type vicinal surfaces and beyond the (443) plane, where we also detect the build-up of surface and/or subsurface oxides. All such features agree with the $\alpha$-dependent variation of the CO chemisorption energy, as well as with the structural transformation of the surface, likely oxygen-induced faceting, beyond critical vicinal angles.

\section{Acknowledgement}

We acknowledge financial support from the Spanish Ministry of Economy (Grants MAT2012-38567-C02-02, MAT-2016-78293-C6, MAT-2017-88374-P) and Basque Government (Grant IT-1255-19). We thank the support of ALBA synchrotron staff for the successful performance of the CIRCE beam line during NAP-XPS experiments.

\section{Supporting Information Available}

Supplementary information and data are provided for UHV experiments performed using the same curved $\operatorname{Pd}(111)$ sample, for a simultaneous gas analysis/XPS scan during the ignition 
process, and for the $\mathrm{Pd} 3 d_{5 / 2}$ peak fits at different temperatures in stationary conditions.

\section{References}

(1) Somorjai, G.; Li, Y. Introduction to Surface Chemistry and Catalysis; John Wiley \& Sons, 2010.

(2) Campbell, C.; Ertl, G.; Kuipers, H.; Segner, J. A molecular beam study of the adsorption and desorption of oxygen from a Pt(111) surface. Surface Science 1981, 10\%, 220 $-236$.

(3) Wintterlin, J.; Völkening, S.; Janssens, T. V. W.; Zambelli, T.; Ertl, G. Atomic and Macroscopic Reaction Rates of a Surface-Catalyzed Reaction. Science 1997, 278, 19311934.

(4) Hendriksen, B.; Bobaru, S.; Frenken, J. Oscillatory CO oxidation on $\operatorname{Pd}(100)$ studied with in situ scanning tunneling microscopy. Surface Science 2004, 552, 229 - 242.

(5) van Spronsen, M. A.; Frenken, J. W. M.; Groot, I. M. N. Surface science under reaction conditions: CO oxidation on Pt and Pd model catalysts. Chem. Soc. Rev. 2017, 46, $4347-4374$.

(6) Ketteler, G.; Ogletree, D. F.; Bluhm, H.; Liu, H.; Hebenstreit, E. L. D.; Salmeron, M. In Situ Spectroscopic Study of the Oxidation and Reduction of Pd(111). Journal of the American Chemical Society 2005, 127, 18269-18273.

(7) van Rijn, R.; Balmes, O.; Resta, A.; Wermeille, D.; Westerström, R.; Gustafson, J.; Felici, R.; Lundgren, E.; Frenken, J. W. M. Surface structure and reactivity of Pd(100) during CO oxidation near ambient pressures. Phys. Chem. Chem. Phys. 2011, 13, $13167-13171$. 
(8) Blomberg, S.; Hoffmann, M. J.; Gustafson, J.; Martin, N. M.; Fernandes, V. R.; Borg, A.; Liu, Z.; Chang, R.; Matera, S.; Reuter, K.; Lundgren, E. In Situ X-Ray Photoelectron Spectroscopy of Model Catalysts: At the Edge of the Gap. Phys. Rev. Lett. 2013, 110, 117601.

(9) Gao, F.; McClure, S.; Cai, Y.; Gath, K.; Wang, Y.; Chen, M.; Guo, Q.; Goodman, D. CO oxidation trends on Pt-group metals from ultrahigh vacuum to near atmospheric pressures: A combined in situ PM-IRAS and reaction kinetics study. Surface Science 2009, 603, $65-70$.

(10) Gao, F.; Wang, Y.; Cai, Y.; Goodman, D. W. CO Oxidation on Pt-Group Metals from Ultrahigh Vacuum to Near Atmospheric Pressures. 2. Palladium and Platinum. The Journal of Physical Chemistry C 2009, 113, 174-181.

(11) Gao, F.; Cai, Y.; Gath, K. K.; Wang, Y.; Chen, M. S.; Guo, Q. L.; Goodman, D. W. CO Oxidation on Pt-Group Metals from Ultrahigh Vacuum to Near Atmospheric Pressures. 1. Rhodium. The Journal of Physical Chemistry C 2009, 113, 182-192.

(12) Gao, F.; Goodman, D. W. Reaction Kinetics and Polarization Modulation Infrared Reflection Absorption Spectroscopy Investigations of CO Oxidation over Planar PtGroup Model Catalysts. Langmuir 2010, 26, 16540-16551.

(13) Weaver, J. F.; Choi, J.; Mehar, V.; Wu, C. Kinetic Coupling among Metal and Oxide Phases during CO Oxidation on Partially Reduced PdO(101): Influence of Gas-Phase Composition. ACS Catalysis 2017, 7, 7319-7331.

(14) Ackermann, M. D.; Pedersen, T. M.; Hendriksen, B. L. M.; Robach, O.; Bobaru, S. C.; Popa, I.; Quiros, C.; Kim, H.; Hammer, B.; Ferrer, S.; Frenken, J. W. M. Structure and Reactivity of Surface Oxides on Pt(110) during Catalytic CO Oxidation. Phys. Rev. Lett. 2005, 95, 255505. 
(15) Westerström, R.; Gustafson, J.; Resta, A.; Mikkelsen, A.; Andersen, J. N.; Lundgren, E.; Seriani, N.; Mittendorfer, F.; Schmid, M.; Klikovits, J.; Varga, P.; Ackermann, M. D.; Frenken, J. W. M.; Kasper, N.; Stierle, A. Oxidation of Pd(553): From ultrahigh vacuum to atmospheric pressure. Phys. Rev. B 2007, 76, 155410.

(16) Gustafson, J.; Westerström, R.; Mikkelsen, A.; Torrelles, X.; Balmes, O.; Bovet, N.; Andersen, J. N.; Baddeley, C. J.; Lundgren, E. Sensitivity of catalysis to surface structure: The example of $\mathrm{CO}$ oxidation on $\mathrm{Rh}$ under realistic conditions. Phys. Rev. B 2008, 78, 045423.

(17) Over, H.; Balmes, O.; Lundgren, E. In situ structure-activity correlation experiments of the ruthenium catalyzed CO oxidation reaction. Catalysis Today 2009, 145, 236 242.

(18) Gustafson, J.; Westerström, R.; Balmes, O.; Resta, A.; van Rijn, R.; Torrelles, X.; Herbschleb, C. T.; Frenken, J. W. M.; Lundgren, E. Catalytic Activity of the Rh Surface Oxide: CO Oxidation over Rh(111) under Realistic Conditions. The Journal of Physical Chemistry C 2010, 114, 4580-4583.

(19) Vlad, A.; Stierle, A.; Westerström, R.; Blomberg, S.; Mikkelsen, A.; Lundgren, E. Oxygen interaction with the $\operatorname{Pd}(112)$ surface: From chemisorption to bulk oxide formation. Phys. Rev. B 2012, 86, 035407.

(20) Farkas, A.; Zalewska-Wierzbicka, K.; Bachmann, C.; Goritzka, J.; Langsdorf, D.; Balmes, O.; Janek, J.; Over, H. High Pressure Carbon Monoxide Oxidation over Platinum (111). The Journal of Physical Chemistry C 2013, 117, 9932-9942.

(21) Gustafson, J.; Shipilin, M.; Zhang, C.; Stierle, A.; Hejral, U.; Ruett, U.; Gutowski, O.; Carlsson, P.-A.; Skoglundh, M.; Lundgren, E. High-Energy Surface X-ray Diffraction for Fast Surface Structure Determination. Science 2014, 343, 758-761. 
(22) Shipilin, M.; Hejral, U.; Lundgren, E.; Merte, L. R.; Zhang, C.; Stierle, A.; Ruett, U.; Gutowski, O.; Skoglundh, M.; Carlsson, P.-A.; Gustafson, J. Quantitative surface structure determination using in situ high-energy SXRD: Surface oxide formation on $\operatorname{Pd}(100)$ during catalytic CO oxidation. Surface Science 2014, 630, 229 - 235.

(23) Shipilin, M.; Gustafson, J.; Zhang, C.; Merte, L. R.; Lundgren, E. Step dynamics and oxide formation during $\mathrm{CO}$ oxidation over a vicinal Pd surface. Phys. Chem. Chem. Phys. 2016, 18, 20312-20320.

(24) Toyoshima, R.; Yoshida, M.; Monya, Y.; Kousa, Y.; Suzuki, K.; Abe, H.; Mun, B. S.; Mase, K.; Amemiya, K.; Kondoh, H. In Situ Ambient Pressure XPS Study of CO Oxidation Reaction on $\operatorname{Pd}(111)$ Surfaces. The Journal of Physical Chemistry C 2012, $116,18691-18697$.

(25) Zemlyanov, D.; Klötzer, B.; Gabasch, H.; Smeltz, A.; Ribeiro, F. H.; Zafeiratos, S.; Teschner, D.; Schnörch, P.; Vass, E.; Hävecker, M.; Knop-Gericke, A.; Schlögl, R. Kinetics of Palladium Oxidation in the mbar Pressure Range: Ambient Pressure XPS Study. Topics in Catalysis 2013, 56, 885-895.

(26) Toyoshima, R.; Yoshida, M.; Monya, Y.; Suzuki, K.; Amemiya, K.; Mase, K.; Mun, B. S.; Kondoh, H. In Situ Photoemission Observation of Catalytic CO Oxidation Reaction on $\mathrm{Pd}(110)$ under Near-Ambient Pressure Conditions: Evidence for the Langmuir-Hinshelwood Mechanism. The Journal of Physical Chemistry C 2013, 117, $20617-20624$.

(27) Wrobel, R. J.; Becker, S.; Weiss, H. Influence of Subsurface Oxygen in the Catalytic CO Oxidation on $\mathrm{Pd}(111)$. The Journal of Physical Chemistry C 2015, 119, 5386-5394.

(28) Gustafson, J.; Balmes, O.; Zhang, C.; Shipilin, M.; Schaefer, A.; Hagman, B.; Merte, L. R.; Martin, N. M.; Carlsson, P.-A.; Jankowski, M.; Crumlin, E. J.; Lund- 
gren, E. The Role of Oxides in Catalytic CO Oxidation over Rhodium and Palladium. ACS Catalysis 2018, 8, 4438-4445.

(29) Vendelbo, S. B.; Elkjaer, C. F.; Falsig, H.; Puspitasari, I.; Dona, P.; Mele, L.; Morana, B.; Nelissen, B. J.; van Rijn, R.; Creemer, J. F.; Kooyman, P. J.; Helveg, S. Visualization of oscillatory behaviour of Pt nanoparticles catalysing CO oxidation. Nat Mater 2014, 13, 884-890.

(30) Tao, F. F.; Salmeron, M. In Situ Studies of Chemistry and Structure of Materials in Reactive Environments. Science 2011, 331, 171-174.

(31) Dou, J.; Sun, Z.; Opalade, A. A.; Wang, N.; Fu, W.; Tao, F. F. Operando chemistry of catalyst surfaces during catalysis. Chem. Soc. Rev. 2017, 46, 2001-2027.

(32) Walter, A. L.; Schiller, F.; Corso, M.; Merte, L. R.; Bertram, F.; Lobo-Checa, J.; Shipilin, M.; Gustafson, J.; Lundgren, E.; Brión-Ríos, A. X.; Cabrera-Sanfelix, P.; Sánchez-Portal, D.; Ortega, J. E. X-ray photoemission analysis of clean and carbon monoxide-chemisorbed platinum(111) stepped surfaces using a curved crystal. Nature Communications 2015, 6, 8903.

(33) Sander, M.; Imbihl, R.; Ertl, G. Kinetic oscillations in catalytic CO oxidation on a cylindrical Pt single crystal surface. The Journal of Chemical Physics 1992, 97, 51935204 .

(34) Calderón, S. K.; Grabau, M.; Óvári, L.; Kress, B.; Steinrück, H.-P.; Papp, C. CO oxidation on Pt(111) at near ambient pressures. The Journal of Chemical Physics 2016, 144,044706 .

(35) Ogletree, D. F.; Bluhm, H.; Lebedev, G.; Fadley, C. S.; Hussain, Z.; Salmeron, M. A differentially pumped electrostatic lens system for photoemission studies in the millibar range. Review of Scientific Instruments 2002, 73, 3872-3877. 
(36) Bluhm, H.; Hävecker, M.; Knop-Gericke, A.; Kiskinova, M.; Schlögl, R.; Salmeron, M. In Situ X-Ray Photoelectron Spectroscopy Studies of Gas-Solid Interfaces at NearAmbient Conditions. MRS Bulletin 2007, 32, 1022-1030.

(37) Doniach, S.; Šunjic, M. Many-electron singularity in X-ray photoemission and X-ray line spectra from metals. Journal of Physics C: Solid State Physics 1970, 3, 285.

(38) The restriction to a minimum number of lines required assymetric Donjiac- Sunjic functions in all lines. For chemisorbed and oxide species the assymetric tail can be justified from the intimate contact with the metal substrate or the presence of vibronic excitations. We can neither discard the contribution of highly dense oxygen and CO chemisorbed phases.

(39) Surnev, S.; Sock, M.; Ramsey, M.; Netzer, F.; Wiklund, M.; Borg, M.; Andersen, J. CO adsorption on $\operatorname{Pd}(111)$ : a high-resolution core level photoemission and electron energy loss spectroscopy study. Surface Science 2000, 470, $171-185$.

(40) Martin, N. M.; Van den Bossche, M.; Grönbeck, H.; Hakanoglu, C.; Zhang, F.; Li, T.; Gustafson, J.; Weaver, J. F.; Lundgren, E. CO Adsorption on Clean and Oxidized Pd(111). The Journal of Physical Chemistry C 2014, 118, 1118-1128.

(41) Blomberg, S.; Zetterberg, J.; Zhou, J.; Merte, L. R.; Gustafson, J.; Shipilin, M.; Trinchero, A.; Miccio, L. A.; Magaña, A.; Ilyn, M.; Schiller, F.; Ortega, J. E.; Bertram, F.; Grönbeck, H.; Lundgren, E. Strain Dependent Light-off Temperature in Catalysis Revealed by Planar Laser-Induced Fluorescence. ACS Catalysis 2017, 7, $110-114$.

(42) For the gas phase peaks, the slight intensity decrease from A to B only reflects a varying surface/gas sensitivity caused by the non-constant emission geometry (see Methods).

(43) Besocke, K.; Krahl-Urban, B.; Wagner, H. Dipole moments associated with edge atoms; 
A comparative study on stepped Pt, Au and W surfaces. Surface Science 1977, 68, 39 $-46$.

(44) Poelsema, B.; Palmer, R. L.; Comsa, G. Helium scattering and work function investigation of co adsorption on Pt(111) and vicinal surfaces. Surface Science 1982, 123, $152-164$.

(45) Johansson, N.; Andersen, M.; Monya, Y.; Andersen, J. N.; Kondoh, H.; Schnadt, J.; Knudsen, J. Ambient pressure phase transitions over $\operatorname{Ir}(111)$ : at the onset of CO oxidation. Journal of Physics: Condensed Matter 2017, 29, 444002.

(46) Hammer, B.; Morikawa, Y.; Nørskov, J. K. CO Chemisorption at Metal Surfaces and Overlayers. Phys. Rev. Lett. 1996, 76, 2141-2144.

(47) Abild-Pedersen, F.; Andersson, M. CO adsorption energies on metals with correction for high coordination adsorption sites - A density functional study. Surface Science 2007, 601, $1747-1753$.

(48) Lauterbach, J.; Boyle, R.; Schick, M.; Mitchell, W.; Meng, B.; Weinberg, W. The adsorption of CO on $\operatorname{Ir}(111)$ investigated with FT-IRAS. Surface Science 1996, 350, $32-44$.

(49) Jeroro, E.; Hyman, M. P.; Vohs, J. M. Ensemble vs. electronic effects on the reactivity of two-dimensional Pd alloys: a comparison of $\mathrm{CO}$ and $\mathrm{CH} 3 \mathrm{OH}$ adsorption on $\mathrm{Zn} / \mathrm{Pd}(111)$ and $\mathrm{Cu} / \mathrm{Pd}(111)$. Phys. Chem. Chem. Phys. 2009, 11, 10457-10465.

(50) Tränkenschuh, B.; Fritsche, N.; Fuhrmann, T.; Papp, C.; Zhu, J. F.; Denecke, R.; Steinrück, H.-P. A site-selective in situ study of CO adsorption and desorption on Pt(355). The Journal of Chemical Physics 2006, 124, 074712.

(51) Rose, M.; Mitsui, T.; Dunphy, J.; Borg, A.; Ogletree, D.; Salmeron, M.; Sautet, P. 
Ordered structures of CO on Pd(111) studied by STM. Surface Science 2002, 512, 48 $-60$.

(52) Zemlyanov, D.; Klötzer, B.; Gabasch, H.; Smeltz, A.; Ribeiro, F. H.; Zafeiratos, S.; Teschner, D.; Schnörch, P.; Vass, E.; Hävecker, M.; Knop-Gericke, A.; Schlögl, R. Kinetics of Palladium Oxidation in the mbar Pressure Range: Ambient Pressure XPS Study. Topics in Catalysis 2013, 56, 885-895.

(53) Ketteler, G.; Ogletree, D. F.; Bluhm, H.; Liu, H.; Hebenstreit, E. L. D.; Salmeron, M. In Situ Spectroscopic Study of the Oxidation and Reduction of Pd(111). Journal of the American Chemical Society 2005, 127, 18269-18273. 
Graphical TOC Entry

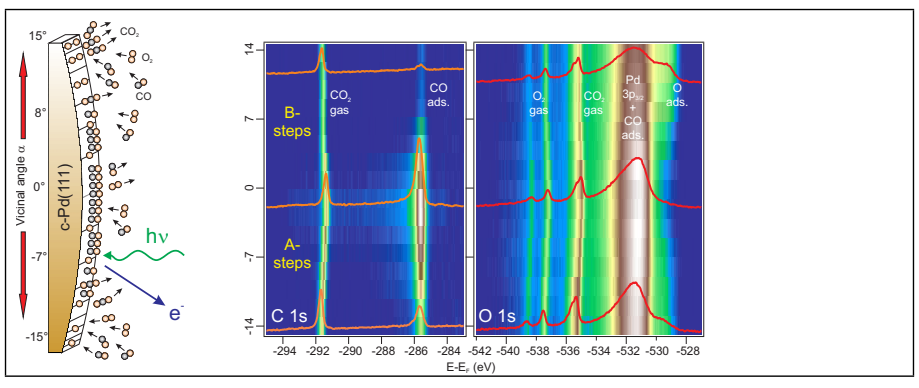

